\title{
Smartphone Applications for Reproduction: From Rigorously Validated and Clinically Relevant to Potentially Harmful
}

\author{
Author: \\ Carol Lynn Curchoe \\ Colorado Center for Reproductive Medicine, Newport Beach, California, USA \\ *Correspondence to cburton@fertilitylabsciences.com \\ Disclosure: $\quad$ Dr Curchoe reports an affiliation as the Founder of ART Compass, \\ and as an expert on the MedAnswers platform. \\ Received: \\ 10.02 .20 \\ Accepted: \\ 16.03 .20 \\ Keywords: \\ Fertility, infertility, mobile applications (apps), quality control, reproduction. \\ Citation: \\ EMJ Repro Health. 2020;6[1]:85-91
}

\section{Abstract}

Infertility practitioners are increasingly turning to mobile applications (apps) to help improve patient care. Provider-facing apps range from reference to communication tools, to versions of the electronic health record. Some available evidence indicates that mobile apps facilitate patient care by increasing efficiency and accuracy in documentation, information retrieval, and coordination of care.' The U.S. Food and Drug Administration (FDA) website currently lists dozens of cleared or approved applications for mobile 'medical device' apps, and reproductive healthcare has also certainly benefitted from this explosion in mobile technology. Reproductive and fertility-focussed apps now aim to treat and diagnose disease, aid clinical decision-making, and manage patient care. Infertility patients may use apps pretreatment to manage lifestyle factors, during treatment to manage medications and calendar appointments, and for message boards where they can share experiences, and seek or offer peer support. Here, the authors review the history of medical health and research apps, current reproduction-specific mobile applications, and discuss the implications of mobile technology for diagnostic point-of-care, clinical research, and patient health management.

\section{INTRODUCTION}

Mobile device applications (apps) debuted in 2008, and the world was transformed by these small pieces of software, seemingly overnight. Medical science has been no exception. Over 165,000 health-related apps now improve health, facilitate clinical trials research, and most recently, integrate with sophisticated external hardware, such as microfluidic chambers, to deliver pointof-care diagnostic assays. It has been estimated that at least 500 million smartphone owners

use a healthcare app; however, a challenge for any new technology is proving that it meets the accuracy standards of tested, tried, and true methods. New health or research related apps require vigorous validation. This is of great importance to the field of reproduction, where patients are using apps to crowdsource tips on improving fertility and preventing pregnancy, chart cycles by last menstrual period date, time intercourse, track and chart medical information (including cervical fluid, basal body temperature, and fertility medications), and to seek support and share experiences. For this reason, the FDA 
issued oversight guidance in 2013 for apps that present a risk to patients if they do not work as intended, and for apps that allow mobile devices to impact the functionality or performance of a traditional medical device. The FDA website lists dozens of cleared or approved applications for mobile medical device apps. ${ }^{7}$

Apple supports the medical community with three products: ResearchKit, HealthKit, and CareKit. These are free, 'open source' resources that allow any app developer to download standard modules from Apple. Generally, CareKit provides tools that lets users regularly track care plans, monitor their progress, and share their insights with care teams. HealthKit allows health and activity data to be tracked and communicated to other apps. ResearchKit allows researchers to conduct clinical studies quickly, cheaply, and easily by collecting survey data through a dedicated study app on the participant's iPhone. These patient-facing apps have harnessed proprietary mobile hardware, (Global Positioning System, accelerometer, gyroscope, touch screen, and microphone) to take and record biometric data.

ResearchKit facilitates Health Insurance Portability and Accountability Act (HIPAA) compliant research projects ${ }^{3}$ in disease areas such as asthma, Parkinson's, ${ }^{4}$ diabetes, melanoma, ${ }^{5}$ and breast cancer, anterior cruciate ligament $(A C L)$ rupture, ${ }^{6}$ and cardiovascular disease, ${ }^{7}$ but peer-reviewed validation studies have been scarce. The Icahn School of Medicine at Mount Sinai Asthma Mobile Health Study, ${ }^{8}$ powered by the ResearchKitlinked iPhone app Asthma Health, has published peer-reviewed survey results in line with existing 'gold standard' research on asthma patients, and rigorous evidence of the app's utility.

Increasingly, smartphone-based medical apps have used sophisticated external hardware addons to perform everything from enzyme-linked immunosorbent assay (ELISA) assays, ${ }^{9,10}$ to ultrasonography, ${ }^{11}$ parasitic microscopy, ${ }^{12}$ and quantum dot barcoding for rapid viral detection. ${ }^{13}$ However, these devices and apps tend to be targeted to resource-poor geographical locations, designed to be deployed to, and have specimens collected by, laboratory technicians, with the results intended to be read and interpreted by healthcare professionals before communication to the patient.
On $10^{\text {th }}$ September 2019, Apple made another major announcement that extended its support of the medical research community. They announced three 'unprecedented' medical studies, in partnership with leading academic and research institutions that will be available on the new Research app, which claim to "democratizes how medical research is conducted by bringing together academic medical institutions, healthcare organizations and the Apple products customers already make a part of their everyday life." Along with a comprehensive heart/movement study and a hearing study, Apple announced a major fertility initiative.

\section{From Apple's press release:}

"Apple Women's Health Study: In partnership with Harvard T.H. Chan School of Public Health and the NIH's National Institute of Environmental Health Sciences (NIEHS), Apple has initiated the first long-term study of this scale focused on menstrual cycles and gynecological conditions. This study will inform screening and risk assessment of conditions like polycystic ovary syndrome (PCOS), infertility, osteoporosis, pregnancy and menopausal transition." ${ }^{14}$

The last study is of particular interest to the field of reproduction, where the use of apps is a burgeoning trend. There are currently over 100 fertility awareness mobile apps ${ }^{15}$ with more than 200 million downloads, being used for contraception and pregnancy planning. Excitingly, fertility awareness apps are just scratching the surface of what is possible in reproductive mobile healthcare.

\section{MOBILE APPLICATIONS FOR REPRODUCTION}

Reproduction apps touch every aspect of the field: diagnostics, clinical research, patient communication and education, embryology laboratory quality control, and medical education. Specific reproduction apps are reviewed in the following section (Table 1).

\section{Diagnostic/Point-of-Care}

In one of the first examples of its kind for reproduction, a sophisticated external microfluidics hardware add-on has been paired with a consumer-facing (no clinical background needed) 
application for diagnostic semen analysis. ${ }^{16}$ The results were published in a rigorously validated and peer-reviewed study using standard World Health Organization (WHO) criteria $^{3}$ for sperm concentration, motility, total sperm count, total motile sperm count, and linear and curvilinear velocities and benchmarked results against gold standard computer-assisted semen analyses (CASA). Additionally, the usability and simplicity of the app was evaluated by recruiting untrained users to participate in a double-blinded evaluation of semen analysis using the smartphone-based platform and CASA. This application was not commercialised at the time of writing this review.

Subsequently, a similar commercially available application called "YO" was FDA approved for motile sperm testing and concentration. It has a cut off value above and below a 6 million per $\mathrm{mL}$, therefore it is not recommended for specialised tests, like vasectomy confirmation. The YO device demonstrated good correlation and good to moderate agreement with an automated semen analyser. The precision among the YO phone devices was lower (16.0\%) than the manufacturer's claim of $\leq 20.0 \% .^{17}$

\section{Clinical Reproduction Research}

Pregnant women have been under-represented in research studies, resulting in dangerous deficiencies in evidence-based guidance for treatment. To that end, Topol et al. ${ }^{18}$ implemented the first pregnancy research app for large-scale collection of survey and sensor-generated data to improve our understanding of factors that promote a healthy pregnancy (for both the mother and developing fetus). They enrolled 2,058 demographically diverse pregnant women from all 50 states, fairly representing USA population averages. They summarised the findings from 14,045 individual surveys and 107,102 total daily measurements of sleep, activity, blood pressure, and heart rate, demonstrating the potential for a smartphone-based research platform to capture an array of longitudinal, objective, and subjective participant-generated data from an under-represented and diverse population of pregnant women.

Table 1: Uses of mobile applications in reproduction.

\begin{tabular}{|c|c|c|c|c|c|}
\hline Mobile apps & $\begin{array}{l}\text { Diagnostic/point } \\
\text { of care }\end{array}$ & Clinical research & Infertility patient & Quality control & $\begin{array}{l}\text { Medical } \\
\text { education }\end{array}$ \\
\hline YO Sperm Test & $\begin{array}{l}\text { Sperm } \\
\text { motility and } \\
\text { concentration }\end{array}$ & & & & \\
\hline Premom & LH test & & & & \\
\hline Healthy Pregnancy & & $\begin{array}{l}\text { Healthy pregnancy } \\
\text { characteristics }\end{array}$ & & & \\
\hline Natural Cycles & & Stages of ovulation & & & \\
\hline SART & & & $\begin{array}{l}\text { IVF clinics } \\
\text { and treatment } \\
\text { options }\end{array}$ & & \\
\hline IVF Planner & & & $\begin{array}{l}\text { Medications, } \\
\text { appointments, } \\
\text { symptoms }\end{array}$ & & \\
\hline IVFqc & & & & Instruments & \\
\hline ART Compass & & & & Staff competency & \\
\hline Embryo App & & & & & $\begin{array}{l}\text { Developmental } \\
\text { biology }\end{array}$ \\
\hline
\end{tabular}

Selected mobile applications in several use categories.

ART: assisted reproductive technology; LH: luteinising hormone; SART: The Society for Assisted Reproductive Technology. 
Bull et al. ${ }^{19}$ analysed over half a million ovulation cycles worth of data collected via the FDAapproved 'Natural Cycles' app to rewrite understanding of the key stages of ovulation. They showed that few women have the textbook 28-day cycle, with some experiencing very short or very long cycles. The findings show an average cycle length is 29.3 days and only around $13 \%$ of cycles are 28 days in length. Across the study, only $65 \%$ of women had cycles that lasted between 25 and 30 days. The Natural Cycles app claims to be useful as a hormone-free method of birth control, studies have demonstrated a 'typical use' failure rate over 13 menstrual cycles of $8.3 \% .^{20}$

\section{Infertility Patient Awareness and Education}

The Society for Assisted Reproductive Technology (SART) recently launched the SART Mobile App. ${ }^{21}$ The app includes a calculator that offers personalised information based on years of national research data and millions of patients and treatments. IVF patients can submit their individual information and receive feedback for various treatment options. They can find and speak with a fertility clinic, schedule clinic appointments, and even manage treatment information. The app also includes a pregnancy wheel and a section for IVF news and information.

An additional 25 apps out of 140 reviewed (17.9\%) contain information or functions specifically related to infertility or its management. ${ }^{22}$ High quality infertility applications were noted as allowing users to track fertility medications, symptoms, and results. Additional features include reminders of fertility doctor appointments and when to administer fertility medication, results tracking (including blood type information, sperm counts, and blood levels), notes section for tracking of issues for later reference, and ability to track symptoms.

\section{IVF Laboratory Digital Quality Control}

There are at least 200 known variables that can impact IVF outcome. ${ }^{23}$ The potential linking of quality control performance to enhanced patient care and outcomes using mobile app technology is intriguing. IVFqc recently released an app called Reflections ${ }^{\mathrm{TM}}$ that allows customisation of quality control for any laboratory instrument and associated parameters. ${ }^{24}$ The app is accessible from any internet-connected device (computer, tablet, or smartphone of any make) and allows for real-time instrument fluctuation tracking and detailed reporting. ${ }^{25}$ They recently reported quality control data from 36 clinics across 12 countries. ${ }^{26}$

In the USA, competency of personnel responsible for testing in an IVF lab is required to be evaluated at least semiannually during the first year the individual tests patient specimens, and at least annually thereafter. Competency assessment must be performed for testing personnel for each test that the individual is approved by the laboratory director to perform. Curchoe et al. ${ }^{27}$ created a HIPAA compliant mobile application ${ }^{28}$ to assess the clinical decision-making of ART laboratory staff for more than 80 common andrology and embryology procedures, track new staff competency during employee on-boarding and annually thereafter, and provide real time in-cycle statistics for staff-related IVF cycle parameters.

Eggschain $^{29}$ is a blockchain-based mobile app that allows women to create a digital, trackable identity using blockchain technology for their frozen eggs and embryos. Eggschain aims to utilise the "immutable and secure" nature of blockchain technology to help women to indisputably prove that frozen eggs and embryos are their own. To date, there have been no abstracts or primary literature published evaluating these claims. ${ }^{29}$

\section{Embryologist Education Applications}

The Carnegie Collection of Embryology, housed at the National Museum of Health and Medicine (NMHM), Silver Spring, Maryland, USA, is used by embryologists worldwide to define normal human embryo development. The Embryo App includes films on fertilisation and IVF, a pregnancy calculator, and a lab manual section. The lab manual presents photographs, 3D reconstructions, animations, reference labels, and information on the early stages (1-23) of embryonic development. The application links to social networks and to extended resources from the National Library of Medicine, Bethesda, Maryland, USA; Louisiana State University, Baton Rouge, Louisiana, USA; NMHM and other institutions. ${ }^{30}$ 


\section{DISCUSSION}

Mobile apps are increasingly used in reproductive healthcare to promote wellness, treat, diagnose, aid clinical decision-making, manage patient care, and collect data for medical research. Mobile app technology has many advantages: HIPAA compliance, rapid data collection and real time reporting, real-time analysis, management and distribution of multi-media files, and the ability to utilise hardware add-ons or proprietary device hardware features, and to collect biometric data to ensure the identity of the tester. However, questions remain about the clinical validity and utility of these new mobile tools for reproductive healthcare. Known limitations of mobile apps include: lack of evidence of clinical effectiveness, lack of integration with the healthcare delivery systems, lack of formal evaluation and review, and potential threats to safety and privacy. ${ }^{31}$

Quality assessment methodologies and tools for mobile apps have been adapted from assessments for other digital technologies, such as websites. Nouri et al. ${ }^{32}$ reviewed currently used models, codes, and scales for assessment of mobile health-related apps. Important assessment criteria included: accuracy, information quality, and security among others. Menstrual tracking apps have been consistently assessed for their functionality and accuracy. In 2016, Moglia et al. $^{33}$ scored 108 menstrual tracking apps, and their primary criterion for ongoing inclusion was accuracy. They concluded "Most free smartphone menstrual cycle tracking apps for patient use are inaccurate. Few cite medical literature or health professional involvement." 33 Updating this analysis in 2019, Zwingerman et al. ${ }^{22}$ identified 140 menstrual tracking apps, with a low overall app quality score of $32 \%$, and a further 31 apps (22.1\%) with serious inaccuracies in content, tools, or both. When 218 menstrual tracking apps were assessed in 2016 for their use in preventing unintended pregnancy, over $40 \%$ were found to not mention any modern contraceptive methods at all. ${ }^{34}$ This systematic review by Mangone et al. ${ }^{34}$ found that very few fertility awareness apps have clinically relevant, evidence-based usefulness, and many of them may even increase the likelihood of unintended pregnancy due to the low effectiveness of the contraceptive methods promoted. ${ }^{34}$ For this reason, the American College of Obstetricians and Gynecologists (ACOG) only advocates use of mobile applications to track menstrual cycles, not as a primary tool to prevent or achieve pregnancy. ${ }^{33}$

The Natural Cycles mobile app investigated by Bull et al. ${ }^{18}$ has a 'typical use' failure rate of preventing pregnancy up to $8.3 \%$ of the time. $^{19}$ In some cases, the failure of the app to prevent pregnancy has resulted in pregnancy-terminations, generating lawsuits in their wake. ${ }^{35}$ The findings of Bull et al. have significant implications for fertility awareness apps that use 'ideal' cycle calendars to generically calculate fertile windows to either plan or prevent a pregnancy. Additionally, there have been major HIPAA compliance concerns with some fertility tracking apps. These applications often ask for intimate details: sexual activity, history of abortions, cervical mucus consistency, orgasm frequency, and preferred sex positions. It was recently reported that the Glow (a pregnancy planning app) was plagued by a series of security flaws, exposing sensitive information to anyone who cared to look. It was characterised as a "jackpot for stalkers." 36 They have since added a new section to their website, inviting hackers to "research" security flaws and responsibly report them.

Without question, laboratory quality control and assurance must be performed routinely in an IVF lab. While the embryologist's role in achieving and contributing to quality ${ }^{37}$ through safety in the assisted reproduction lab is obvious; appropriate levels of monitoring, what to monitor, and the best ways to monitor it are surprisingly unclear. Until just recently, the anatomy of a liquid nitrogen dewar failure, ${ }^{38}$ how a storage vessel behaves when the vacuum is breached, was unknown, and recent investigations have also quantified major differences in instrument monitoring practices worldwide. ${ }^{26}$ Staff competency is a crucial component of the IVF laboratory's quality management system because it directly impacts clinical outcomes. Embryologists must be competent to make several clinical decisions that can affect cycle outcomes. Certain IVF key performance indicators ${ }^{39}$ are used to continuously monitor and assess culture conditions.

Digital and 'cloud based' solutions to discover malfunctioning instruments and environments have been best practice tools for over 20 years in other industries such as aerospace, automotive, 
and manufacturing. It seems as though the time of digital quality control is long overdue for the IVF lab, and that IVFqc and ART Compass represent a new paradigm, but future publications detailing how the measured parameters relate to clinical outcomes will help to further advance the field of IVF lab quality control.

\section{Cryopreservation of reproductive tissues was} once an adjunctive procedure, but in contemporary IVF labs it enables preimplantation genetic diagnosis after blastocyst biopsy, deferred transfer to a more favourable uterine environment, and fertility preservation. Chain of custody for reproductive tissues is related to both staff competency and quality control, with cryopreservation and storage of human embryos and gametes emerging as a clear subspecialty of the assisted reproductive technologies industry. ${ }^{40}$ New technologies, including a mobile app that promises to help solve chain of custody issues, such as pairing the wrong gametes together or the wrong embryo with the intended parent, are needed. The Eggschain mobile app, while promising, is marketing a 'clear chain of custody for decisions by-laws, contracts, estate plans.' It is unclear at the time of writing if the technology could fulfill these promises, or if it has been tested in a court of law.

\section{CONCLUSION}

The challenge for these new technologies is to prove that they meet the accuracy standards of tested, tried, and true methods. New health or research-related apps that have the potential to impact patients, curate very sensitive health data, and or impact patient care require vigorous validation.

\section{References}

1. Ventola CL. Mobile devices and apps for health care professionals: uses and benefits. P T. 2014;39(5):356-64.

2. US Food and Drug Administration (FDA). Examples of Pre-Market Submissions that Include MMAs Cleared or Approved by the FDA. 2018. Available at: www. fda.gov/medical-devices/devicesoftware-functions-includingmobile-medical-applications/ examples-premarket-submissionsinclude-mmas-cleared-or-approvedfda. Last accessed: 06.04.2020.

3. Rosa C et al. Using e-technologies in clinical trials. Contemp Clin Trials, 2015;45(PtA):41-54.

4. Bot B et al. The mPower study, Parkinson disease mobile data collected using ResearchKit. Sci Data. 2016;3:160011.

5. Webster D et al. The Mole Mapper Study, mobile phone skin imaging and melanoma risk data collected using ResearchKit. Sci Data. 2017;4:170005.

6. Zens M et al. "Back on Track": a mobile app observational study using Apple's ResearchKit framework. JMIR Mhealth Uhealth. 2017;5(2):e23.

7. McConnell $M$ et al. Feasibility of obtaining measures of lifestyle from a smartphone app: the MyHeart Counts cardiovascular health study. JAMA Cardiol. 2017;2(1):67-76.

8. Chan YF et al. The asthma mobile health study, a large-scale clinical observational study using ResearchKit. Nat Biotechnol.

\section{7;35(4):354-62.}

9. Laksanasopin T et al. A smartphone dongle for diagnosis of infectious diseases at the point of care. Sci Transl Med. 2015;7(273):273re1.

10. Berg B et al. Cellphone-based handheld microplate reader for pointof-care testing of enzyme-linked immunosorbent assays. ACS Nano. 2015;9(8):7857-66

11. Wojtczak J, Bonadonna P. Pocket mobile smartphone system for the point-of-care submandibular ultrasonography. Am J Emerg Med. 2013;31(3):573-7.

12. D'Ambrosio $M$ et al Point-of-care quantification of blood-borne filarial parasites with a mobile phone microscope. Sci Transl Med. 2015;7(286):286re4.

13. Ming $\mathrm{K}$ et al. Integrated quantum dot barcode smartphone optical device for wireless multiplexed diagnosis of infected patients. ACS Nano. 2015;9(3):3060-74.

14. Apple Inc. Apple announces three groundbreaking health studies. 2019. Available from: www.apple. com/newsroom/2019/09/appleannounces-three-groundbreakinghealth-studies/ Last accessed: 07.04.2020.

15. Dreaper J. Women warned about booming market in period tracker apps. 2016. Available at: www.bbc. co.uk/news/health-37013217. Last accessed: 20.03.2020.
16. Kanakasabapathy MK et al. An automated smartphone-based diagnostic assay for point-of-care semen analysis. Sci Transl Med. 2017;9(382).

17. Agarwal A et al. Home sperm testing device versus laboratory sperm quality analyzer: comparison of motile sperm concentration. Fertil Steril. 2018;110(7):1277-84.

18. Radin $\mathrm{J}$ et al. The healthy pregnancy research program: transforming pregnancy research through a ResearchKit app. NPJ Digit Med. 2018; $1: 45$.

19. Bull J et al. Real-world menstrual cycle characteristics of more than 600,000 menstrual cycles. NPJ Digit Med. 2019;2:83.

20. Berglund Scherwitzl E et al. Perfectuse and typical-use Pearl Index of a contraceptive mobile app. Contraception. 2017;96(6):420-5.

21. Society for Assisted Reproductive Technology, Apple Store. SART mobile: the official SART app for IVF. 2020. Available from: https:// apps.apple.com/us/app/sartmobile/id1370121034. Last accessed: 20.03.2020.

22. Zwingerman $\mathrm{R}$ et al. A critical appraisal of fertility and menstrual tracking apps for the iPhone. J Obstet Gynaecol Can. 2019. [ePub ahead of print].

23. Pool T et al. Human embryo culture media comparisons. Methods Mol Biol. 2012;912:367-86. 
24. Althea Science Inc. IVFqc. 2020. Avaiable at: www.ivfqc.com. Last accessed: 20.03 .2020

25. Fiser $S$ et al. IVF data flow: from trash bins to clipboards to EMR to smart apps. Fertility. 2016;19:24-6.

26. Palmer $\mathrm{G}$ et al. Comparison of 36 assisted reproduction laboratories monitoring environmental conditions and instrument parameters using the same quality-control application. Reprod Biomed Online. 2019;39(1):6374.

27. Curchoe $\mathrm{C}$ et al. Development of a mobile competency assessment platform for IVF laboratory quality managment systems. Abstract Foundation for Reproductive Medicine Annual Conference, 15-18 November, 2018

28. ART Compass. ART Compass. Available at: www.artcompass.io. Last accessed: 20.03.2020

29. Alice. Eggschain is empowering women who are choosing to freeze their eggs. 2018. Available at: https://medium.com/successfulentrepreneurs-role-breakers/weiescala-eggschain-successful-womenentrepreneurs-68e09ca98659. Last accessed: 20.03.2020.

20.03.2020

30. Haseltine F et al. The Mobile Embryo Application. FASEB J. 2011;25(Suppl 1):Ib12.

31. Carroll J et al. Who uses mobile phone health apps and does use matter? a secondary data analytics approach. J Med Internet Res. 2017;19(4):e125.

32. Nouri R et al. Criteria for assessing the quality of mHealth apps: a systematic review. J Am Med Inform Assoc. 2018;25(8):1089-98.

33. Moglia M et al. Evaluation of smartphone menstrual cycle tracking applications using an adapted APPLICATIONS scoring system. Obstet Gynecol. 2016;127(6):1153-60

34. Mangone E et al. Mobile phone apps for the prevention of unintended pregnancy: a systematic review and content analysis. JMIR Mhealth Uhealth. 2016;4(1):e6

35. Global News. Natural Cycles contraceptive app blamed for 37 unwanted pregnancies. 2018. Available at: https://globalnews.ca/ news/3967648/contaceptive-appunwanted-pregnancy/. Last accessed:
36. Weill K. This fertility app is a jackpot for stalkers. 2017. Available at: https:// www.thedailybeast.com/this-fertilityapp-is-a-jackpot-for-stalkers. Last accessed: 20.03 .2020

37. Go K. 'By the work, one knows the workman': the practice and profession of the embryologist and its translation to quality in the embryology laboratory. Reprod Biomed Online. 2015. 31(4):449-58.

38. Pomeroy $\mathrm{K}$ et al. Cryostorage tank failures: temperature and volume loss over time after induced failure by removal of insulative vacuum. J Assist Reprod Genet. 2019;36(11):2271-8.

39. ESHRE Special Interest Group of Embryology and Alpha Scientists in Reproductive Medicine. The Vienna consensus: report of an expert meeting on the development of ART laboratory performance indicators. Reprod Biomed Online. 2017;35(5):494-510.

40. Go K. A subspecialty of the assisted reproductive technologies: cryogenic inventory maintenance. J Assist Reprod Genet. 2019;36(1):15-17. 